\title{
ASYMPTOTICS FOR ORTHOGONAL RATIONAL FUNCTIONS
}

\author{
A. BULTHEEL, P. GONZALEZ-VERA, E. HENDRIKSEN, AND O. NJASTAD
}

ABSTRACT. Let $\left\{\alpha_{n}\right\}$ be a sequence of (not necessarily distinct) points in the open unit disk, and let

$$
\left.B_{0}=1, \quad B_{n}(z)=\prod_{m=1}^{n} \frac{\overline{\alpha_{m}}}{\left|\alpha_{m}\right|} \frac{\left(\alpha_{m}-z\right)}{\left(1-\overline{\alpha_{m}} z\right.}\right), \quad n=1,2, \ldots,
$$

$\left(\frac{\overline{\alpha_{n}}}{\left|\alpha_{n}\right|}=-1\right.$ when $\left.\alpha_{n}=0\right)$. Let $\mu$ be a finite (positive) Borel measure on the unit circle, and let $\left\{\varphi_{n}(z)\right\}$ be orthonormal functions obtained by orthogonalization of $\left\{B_{n}: n=0,1,2, \ldots\right\}$ with respect to $\mu$. Boundedness and convergence properties of the reciprocal orthogonal functions $\varphi_{n}^{*}(z)=B_{n}(z) \overline{\varphi_{n}(1 / \bar{z})}$ and the reproducing kernels $k_{n}(z, w)=\sum_{m=0}^{n} \varphi_{m}(z) \overline{\varphi_{m}(w)}$ are discussed in the situation $\left|\alpha_{n}\right| \leq R<1$ for all $n$, in particular their relationship to the Szegö condition $\int_{-\pi}^{\pi} \ln \mu^{\prime}(\theta) d \theta>-\infty$ and noncompleteness in $L_{2}(\mu)$ of the system $\left\{\varphi_{n}(z): n=0,1,2, \ldots\right\}$. Limit functions of $\varphi_{n}^{*}(z)$ and $k_{n}(z, w)$ are obtained. In particular, if a subsequence $\left\{\alpha_{n(s)}\right\}$ converge to $\alpha$, then the subsequence $\left\{\varphi_{n(s)}^{*}(z)\right\}$ converges to

$$
e^{i \lambda} \frac{\sqrt{1-|\alpha|^{2}}}{1-\bar{\alpha} z} \frac{1}{\sigma_{\mu}(z)}, \quad \lambda \in \mathbf{R},
$$

where

$$
\sigma_{\mu}(z)=\sqrt{2 \pi} \exp \left[\frac{1}{4 \pi} \int_{-\pi}^{\pi} \frac{e^{i \theta}+z}{e^{i \theta}-z} \ln \mu^{\prime}(\theta) d \theta\right] .
$$

The kernels $\left\{k_{n}(z, w)\right\}$ converge to $1 /(1-z \bar{w}) \sigma_{\mu}(z) \overline{\sigma_{\mu}(w)}$.

The results generalize corresponding results from the classical Szegö theory (concerned with the polynomial situation $\alpha_{n}=0$ for all $n$ ).

\section{INTRODUCTION}

By a Carathéodory function we mean an analytic function in the open unit disk mapping this disk into the open right halfplane. The Carathéodory Coefficient Problem for an infinite sequence $\left\{c_{n}: n=0,1,2, \ldots\right\}$ can be formulated as follows: Find a Carathéodory function whose Taylor coefficients at the origin are the $\left\{c_{n}\right\}$. This problem is equivalent to the Trigonometric Moment Problem: Find a (finite) measure $\mu$ on $[-\pi, \pi]$ such that

$$
\int_{\pi}^{\pi} e^{-i n \theta} d \mu(\theta)=c_{n} \text { for } n=0,1,2, \ldots
$$

Received by the editors June 24, 1993 and, in revised form, April 22, 1994.

1991 Mathematics Subject Classification. Primary 42C05; Secondary 30B70, 30D50, 41A20.

Key words and phrases. Orthogonal rational functions, Szegö theory. 
A measure $\mu$ solves the moment problem if and only if the Carathéodory function

$$
\Omega_{\mu}(z)=\int_{-\pi}^{\pi} \frac{e^{i \theta}+z}{e^{i \theta}-z} d \mu(\theta)
$$

solves the coefficient problem. These problems are again closely related to the theory of Szegö polynomials, i.e., orthonormal polynomials (with respect to $\mu$ ) on the unit circle. In particular, if $\left\{\varphi_{n}^{*}\right\}$ are the reciprocals of the Szegö polynomials $\left\{\varphi_{n}(z)\right\}$ (defined by $\left.\varphi_{n}^{*}(z)=z^{n} \overline{\varphi_{n}(1 / \bar{z})}\right)$ and $\left\{\psi_{n}^{*}\right\}$ are the reciprocals of the associated Szegö polynomials (given by

$$
\left.\psi_{n}(z)=\int_{-\pi}^{\pi}\left[\varphi_{n}\left(e^{i \theta}\right)-\varphi_{n}(z)\right] \frac{d e^{i \theta}+z}{e^{i \theta}-z} d \mu(\theta), \quad \psi_{n}^{*}(z)=z^{n} \psi_{n} \overline{(1 / \bar{z})}\right),
$$

then $\left\{\psi_{n}^{*}(z) / \varphi_{n}^{*}(z)\right\}$ converges to $\Omega_{\mu}(z)$ locally uniformly for $|z|<1$. Furthermore $\left\{1 / \varphi_{n}^{*}(z)\right\}$ converges locally uniformly for $|z|<1$ to the spectral factor

$$
\sigma_{\mu}(z)=\sqrt{2 \pi} \exp \left\{\frac{1}{4 \pi} \int_{-\pi}^{\pi} \frac{e^{i \theta}+z}{e^{i \theta}-z} \ln \mu^{\prime}(\theta) d \theta\right\}
$$

when the measure $\mu$ satisfies Szegö's condition

$$
\int_{-\pi}^{\pi} \ln \mu^{\prime}(\theta) d \theta>-\infty
$$

In addition the reproducing kernel $k_{n}(z, w)$ (given by

$$
\left.k_{n}(z, w)=\sum_{m=0}^{n} \varphi_{n}(z) \overline{\varphi_{n}(w)}\right)
$$

then converges locally uniformly to

$$
s(z, w)=\frac{1}{(1-z \bar{w}) \sigma(z) \overline{\sigma(w)}} .
$$

For more information on these matters we refer to $[1,13,19,20,22,24,26$, $27,29,38]$.

Let $\left\{\alpha_{n}: n=0,1,2, \ldots\right\}$ be an arbitrary sequence of not necessarily distinct points in the open unit disk (for convenience $\alpha_{0}$ is taken to be the origin, which can always be obtained by a simple substitution), and let $\left\{w_{n}: n=\right.$ $0,1,2, \ldots\}$ be a given sequence of complex numbers. The Nevanlinna-Pick Interpolation Problem for this situation is the following: Find a Caratheodory function $F$ with the interpolation property $F\left(\alpha_{n}\right)=w_{n}$ for all $n$. (When points $\alpha_{n}$ are repeated, the interpolation requirement involves the appropriate number of derivatives.) (See $[1,28,32,35]$.) This problem is connected with the problem of finding measures $\mu$ generating generalized moments appearing as coefficients in Newton series expansions for $F(z)$ : A measure $\mu$ generates the moments if and only if the Carathéodory function $\Omega_{\mu}(z)$ defined above solves the interpolation problem. Also these problems are related to a theory of orthogonal functions, here rational functions (with poles at the points $1 / \overline{\alpha_{n}}$ ). The approach to Nevanlinna-Pick theory from the point of view of orthogonal functions is more recent than the study of other aspects of the theory. From a 
purely mathematical point of view the theory of such orthogonal rational functions as far as we now was initiated by Djrbashian about 1960 (see the survey paper [17]). Independently, partly from an applied point of view, the same constructions were used by Bultheel, Bultheel and Dewilde, Dewilde and Dym about 1980 (see $[2,3,16]$ ). General moments of a measure $\mu$ were as far as we know first introduced and used by Lopez (see [30]). Special cases involving cyclic repetition of a finite number of points have later been discussed in detail (see $[5,9,21])$. The groundwork for a systematic general treatment can be found in [4], and the theory has been further developed in $[6,7,8,10,12]$. See also [33, 34] and [31].

Surveys of applications of Szegö theory and Nevanlinna-Pick theory in digital signal processing and circuit theory with references to other work in the same direction can be found in $[14,15]$.

The aim of this paper is to study boundedness and convergence properties of the reciprocal orthogonal functions $\left\{\varphi_{n}^{*}(z)\right\}$ and the reproducing kernels $\left\{k_{n}(z, w)\right\}$. The results generalize corresponding results from the Szegö theory (concerned with the polynomial situation). The investigation is carried through for the situation when all the points $\left\{\alpha_{n}\right\}$ are contained in a compact subset of the open unit disk. Main results are about convergence properties of $\left\{\varphi_{n}^{*}(z)\right\}$ and $\left\{k_{n}(z, w)\right\}$ when the Szegö condition (1.4) is satisfied. In particular, we show that for a subsequence of $\left\{\alpha_{n}\right\}$ converging to $\alpha$, the corresponding subsequence of $\left\{\frac{1}{\varphi_{n}^{*}(z)}\right\}$ converges to

$$
\frac{1}{\pi_{\alpha}(z)}=e^{i \lambda} \frac{1-\bar{\alpha} z}{\sqrt{1-|\alpha|^{2}}} \sigma_{\mu}(z), \quad \lambda \in \mathbf{R},
$$

where $\sigma_{\mu}(z)$ is given by (1.3), and the whole sequence $\left\{k_{n}(z, w)\right\}$ converges to the function $s(z, w)$ given by (1.5). See Theorem 6.12 and Theorem 6.14.

In [4] we proved that $\left\{k_{n}(z, w)\right\}$ converges to $s(z, w)$ in $L_{2}$-norm on $[-\pi, \pi]$ under the weaker restriction (than the one above) $\sum_{n=1}^{\infty}\left(1-\left|\alpha_{n}\right|\right)=\infty$. We also stated the result above about locally uniform convergence in this more general case. We have so far not been able to extend our argument here to the more general situation.

For basic theory of orthogonal rational functions when the interpolation points $\left\{\alpha_{n}\right\}$ lie on $\mathbf{T}$, see [11].

\section{Preliminaries}

We shall use the notation $\mathbf{T}=\{z \in C:|z|=1\}, \mathbf{D}=\{z \in C:|z|<1\}$ for the unit circle and the unit disk. The kernel $D(t, z)$ is defined as

$$
D(t, z)=\frac{t+z}{t-z}
$$

Let $\mu$ be a finite Borel measure on $[-\pi, \pi]$. The Carathéodory function $\Omega_{\mu}$ is defined as the integral transform (Herglotz-Riesz transform)

$$
\Omega_{\mu}(z)=\int_{-\pi}^{\pi} D\left(e^{i \theta}, z\right) d \mu(\theta) .
$$

The substar conjugate $f_{*}$ of a function $f$ is defined by

$$
f_{*}(z)=f \overline{(1 / \bar{z})} .
$$


When $f$ is a rational function, this may be written as

$$
f_{*}(z)=\bar{f}(1 / z),
$$

where the bar denotes complex conjugates of the coefficients. The inner product $\langle$,$\rangle is defined by$

$$
\langle f, g\rangle=\int_{-\pi}^{\pi} f\left(e^{i \theta}\right) \overline{g\left(e^{i \theta}\right)} d \mu(\theta)=\int_{-\pi}^{\pi} f\left(e^{i \theta}\right) g_{*}\left(e^{i \theta}\right) d \mu(\theta) .
$$

For an arbitrary $\alpha$ in $\mathbf{D}$ we define the Blaschke factor $\zeta(\alpha, z)$ by

$$
\zeta(\alpha, z)=\frac{\bar{\alpha}}{|\alpha|} \cdot \frac{(\alpha-z)}{(1-\bar{\alpha} z)} .
$$

(By convention we set $\frac{\bar{\alpha}}{|\alpha|}=-1$ when $\alpha=0$.)

Let $\left\{\alpha_{n}: n=1,2, \ldots\right\}$ be an arbitrary sequence of (not necessarily distinct) points in $\mathbf{D}$. For convenience we use the notation

$$
\zeta_{n}(z)=\zeta\left(\alpha_{n}, z\right)
$$

The Blaschke products $B_{n}$ are defined by

$$
B_{0}(z)=1, \quad B_{n}(z)=\prod_{k=1}^{n} \zeta_{k}(z) \text { for } n=1,2, \ldots
$$

We define the spaces $\mathscr{L}_{n}$ by

$$
\mathscr{L}_{n}=\operatorname{Span}\left\{B_{m}: m=0,1, \ldots, n\right\} .
$$

The functions in $\mathscr{L}_{n}$ are exactly the functions that may be written in the form

$$
L(z)=\frac{p_{n}(z)}{\pi_{n}(z)},
$$

where

$$
\pi_{n}(z)=\prod_{k=1}^{n}\left(1-\overline{\alpha_{k}} z\right), \quad n=1,2, \ldots,
$$

and $p_{n} \in \prod_{n}$ (the space of polynomials of degree at most $n$ ). This follows by partial fraction decomposition. In particular the situation reduces to the polynomial case $\mathscr{L}_{n}=\prod_{n}$ when $\alpha_{n}=0$ for all $n$. For $f \in \mathscr{L}_{n}-\mathscr{L}_{n-1}$ we define its superstar conjugate $f^{*}$ by

$$
f^{*}(z)=B_{n}(z) f_{*}(z) \text {. }
$$

(Note that this transformation depends on $n$. It must be clear from the context what $n$ is.) Observe that $f^{*} \in \mathscr{L}_{n}$ when $f \in \mathscr{L}_{n}$.

Let the sequence $\left\{\Phi_{n}: n=0,1,2, \ldots\right\}$ be obtained by orthogonalization of the sequence $\left\{B_{n}: n=0,2,3, \ldots\right\}$ with respect to the inner product $(2.5)$.

The following orthogonality properties are valid:

$$
\begin{gathered}
\left\langle\Phi_{n}, \Phi_{n}\right\rangle=\left\langle\Phi_{n}^{*}, \Phi_{n}^{*}\right\rangle \neq 0, \\
\left\langle f, \Phi_{n}\right\rangle=0 \text { for } f \in \mathscr{L}_{n-1}, \\
\left\langle f, \Phi_{n}^{*}\right\rangle=0 \text { for } f \in \zeta_{n} \mathscr{L}_{n-1} .
\end{gathered}
$$


(Here $f \in \zeta_{n} \mathscr{L}_{n-1}$ means that $f$ may be written on the form $f(z)=\zeta_{n}(z) g(z)$, where $g \in \mathscr{L}_{n-1}$.)

Each $\Phi_{n}$ has a decomposition

$$
\Phi_{n}(z)=\sum_{k=0}^{n} b_{k}^{(n)} B_{k}(z) .
$$

By calculating $\Phi_{n}^{*}(z)$ and substituting $\alpha_{n}$ for $z$ we verify that

$$
\overline{\Phi_{n}^{*}\left(\alpha_{n}\right)}=b_{n}^{(n)} \text {. }
$$

We shall reserve the notation $\Phi_{n}$ for the monic functions, i.e., those for which $b_{n}^{(n)}=1$. We denote by $\kappa_{n}$ the inverse of the norm of $\Phi_{n}$, i.e.,

$$
\kappa_{n}=\left\langle\Phi_{n}, \Phi_{n}\right\rangle^{-1 / 2} \text {. }
$$

We denote by $\varphi_{n}$ the normalized functions,

$$
\varphi_{n}(z)=\kappa_{n} \Phi_{n}(z) \text {. }
$$

We thus have

$$
\varphi_{n}^{*}\left(\alpha_{n}\right)=\kappa_{n}, \quad \Phi_{n}^{*}\left(\alpha_{n}\right)=1 .
$$

It can be shown that $\varphi_{n}^{*}(z)$ solves the extremal problem

$$
\max \left\{\left|f\left(\alpha_{n}\right)\right|:\langle f, f\rangle=1, f \in \mathscr{L}_{n}\right\} .
$$

(See [4, p. 27].) The sequence $\left\{\mathscr{L}_{n}\right\}$ is nested, i.e., $\mathscr{L}_{n} \subset \mathscr{L}_{n+1}$. It follows that if $\alpha_{n}=\alpha$ for all $n$, then $\left\{\kappa_{n}\right\}=\left\{\varphi_{n}^{*}\left(\alpha_{n}\right)\right\}$ is a nondecreasing sequence. This monotonicity property of $\left\{\kappa_{n}\right\}$ does not follow in general.

The functions $\varphi_{n}$ have all their zeros in $\mathbf{D}$, while the functions $\varphi_{n}^{*}$ have all their zeros in $E=\{z \in \mathbf{C}:|z|>1\}$.

The associated functions $\left\{\psi_{n}\right\}$ are defined by

$$
\begin{array}{r}
\psi_{0}=\frac{1}{\kappa_{0}}, \quad \psi_{n}(z)=\int_{-\pi}^{\pi} D\left(e^{i \theta}, z\right)\left[\varphi_{n}\left(e^{i \theta}\right)-\varphi_{n}(z)\right] d \mu(\theta), \\
n=1,2, \ldots .
\end{array}
$$

Their superstar conjugates $\left\{\psi_{n}^{*}\right\}$ can be written

$$
\begin{aligned}
& \psi_{0}^{*}=\frac{1}{\kappa_{0}}, \quad \psi_{n}^{*}(z)=-\int_{-\pi}^{\pi} D\left(e^{i \theta}, z\right)\left[\frac{B_{n}(z)}{B_{n}\left(e^{i \theta}\right)} \varphi_{n}^{*}\left(e^{i \theta}\right)-\varphi_{n}^{*}(z)\right] d \mu(\theta), \\
& n=1,2, \ldots .
\end{aligned}
$$

The reproducing kernel $k_{n}(z, w)$ for the space $\mathscr{L}_{n}$ is defined by the property

$$
L(w)=\left\langle L, k_{n}\right\rangle=\int_{-\pi}^{\pi} L\left(e^{i \theta}\right) \overline{k_{n}\left(e^{i \theta}, w\right)} d \mu(\theta) \quad \text { for } L \in \mathscr{L}_{n} .
$$

It has the representation

$$
k_{n}(z, w)=\sum_{m=0}^{n} \varphi_{m}(z) \overline{\varphi_{m}(w)}
$$


The following Christoffel-Darboux formula is valid:

$$
k_{n}(z, w)=\frac{\varphi_{n}^{*}(z) \overline{\varphi_{n}^{*}(w)}-\zeta_{n}(z) \overline{\zeta_{n}(w)} \varphi_{n}(z) \overline{\varphi_{n}(w)}}{1-\zeta_{n}(z) \overline{\zeta_{n}(w)}} .
$$

From $(2.25)-(2.26)$ follows

$$
\frac{\varphi_{n}^{*}(z) \overline{\varphi_{n}^{*}(w)}-\zeta_{n}(z) \overline{\zeta_{n}(w)} \varphi_{n}(z) \overline{\varphi_{n}(w)}}{1-\zeta_{n}(z) \overline{\zeta_{n}(w)}}=\sum_{m=0}^{n} \varphi_{m}(z) \overline{\varphi_{m}(w)}
$$

The following interpolation property holds:

$$
\frac{\psi_{n}^{*}(z)}{\varphi_{n}^{*}(z)}-\Omega_{\mu}(z)=B_{n}(z) H_{n}(z)
$$

where $H_{n}(z)$ is analytic in $\mathbf{D}, H_{n}(0)=0$. Furthermore the following convergence property holds:

$$
\left\{\frac{\psi_{n}^{*}(z)}{\varphi_{n}^{*}(z)}\right\} \underset{n \rightarrow \infty}{\longrightarrow} \Omega_{\mu}(z)
$$

locally uniformly on $\mathbf{D}$, if $\sum_{n=1}^{\infty}\left(1-\left|\alpha_{n}\right|\right)=\infty$.

For more details on this introductory material, see $[4,6,10]$.

We easily observe that for given positive numbers $r, R$, both less than 1 , the value of $|\zeta(\alpha, z)|$ has a maximum less than 1 for $|z| \leq r,|\alpha| \leq R$. When nothing else is indicated, we shall in the the following assume that there is a constant $R$ less than 1 such that $\left|\alpha_{n}\right| \leq R$ for all $n$.

We then have

$$
\left|\zeta_{n}(z)\right| \leq m(r)<1
$$

for all $n$ and all $|z| \leq r$.

\section{BOUNDEDNESS AND CONVERGENCE}

We shall in this section discuss in some detail boundedness and convergence properties in $\mathbf{D}$ of the sequences $\left\{\varphi_{n}^{*}\right\}$ and $\left\{\kappa_{n}\right\}$.

Proposition 3.1. Every subsequence $\left\{\varphi_{n(s)}^{*}\right\}$ of $\left\{\varphi_{n}^{*}\right\}$ has a subsequence $\left\{\varphi_{n(s(p))}^{*}\right\}$ which converges locally uniformly to an analytic function $\pi$ without zeros or diverges locally uniformly to $\infty$.

Proof. From (2.27) with $w=z$ follows that

$$
\left|\varphi_{n}^{*}(z)\right|^{2} \geq \kappa_{0}^{2}\left(1-\left|\zeta_{n}(z)\right|^{2}\right) \geq \kappa_{0}^{2}\left(1-m(r)^{2}\right)>0
$$

for $|z| \leq r<1$. The existence of a locally uniformly convergent or locally uniformly divergent subsequence as stated then follows from the theory of normal families (Montel's theorem, see, e.g., [23]). That the limit function $\pi$ has no zeros follows from Hurwitz's theorem, see, e.g., [23], since $\varphi_{n}^{*}$ has no zeros in D.

Proposition 3.2. The sequence $\left\{\varphi_{n}^{*}\right\}$ is either locally uniformly bounded in $\mathbf{D}$ or diverges locally uniformly to $\infty$ in $\mathbf{D}$.

Proof. Assume that $\left\{\varphi_{n}^{*}\right\}$ does not diverge locally uniformly to $\infty$. Then $\left\{\varphi_{n}^{*}\right\}$ has a subsequence containing no subsequence diverging locally uniformly to $\infty$, hence by Proposition 3.1 there exists a subsequence $\left\{\varphi_{n(q)}^{*}\right\}$ which converges 
locally uniformly (to a finite function) in $D$. It follows from (2.27) with $w=z$ that

$$
k_{n(q)}(z, z)=\sum_{m=0}^{n(q)}\left|\varphi_{m}(z)\right|^{2} \leq \frac{\left|\varphi_{n(q)}^{*}(z)\right|^{2}}{1-m(r)^{2}}
$$

for $|z| \leq r<1$. Since $\left\{\varphi_{n(q)}^{*}\right\}$ is locally uniformly bounded and the sequence $\left\{k_{n}(z, z)\right\}$ is nondecreasing (by $(2.25)$ ), it follows that the whole sequence $\left\{k_{n}(z, z)\right\}$ is locally uniformly bounded. Then $\left\{\varphi_{n}\right\}$ is locally uniformly bounded by (2.25), thus $\left\{\left|\varphi_{n}^{*}\right|^{2}\right\}$ and hence $\left\{\varphi_{n}^{*}\right\}$ is locally uniformly bounded by (2.27).

Corollary 3.3. The sequence $\left\{\kappa_{n}\right\}$ is either bounded or diverges to $\infty$.

Proof. Assume that $\left\{\kappa_{n}\right\}$ does not diverge to $\infty$. Since $\kappa_{n}=\varphi_{n}^{*}\left(\alpha_{n}\right)$ this implies that $\left\{\varphi_{n}^{*}\right\}$ does not diverge locally uniformly to $\infty$. Then by Proposition $3.2\left\{\varphi_{n}^{*}\right\}$ is locally uniformly bounded, hence $\left\{\kappa_{n}\right\}$ is bounded.

We shall discuss relationships between various properties connected with the measure $\mu$ and the spaces $\mathscr{L}$. In this section we consider the properties I-IV and $\mathrm{I}^{*}-\mathrm{II}^{*}$ below.

I. The sequence $\left\{\kappa_{n}\right\}$ is bounded.

II. The sequence $\left\{\varphi_{n}^{*}\right\}$ is locally uniformly bounded in $\mathbf{D}$.

III. The series $\left.\sum_{m=0}^{\infty}\left|\varphi_{m}(z)\right|^{2} \varphi_{m}(z)\right|^{2}$ converges locally uniformly in $\mathbf{D}$.

IV. The sequence $\left\{k_{n}(z, w)\right\}$ converges locally uniformly for $z, w \in \mathbf{D}$.

$I^{*}$. The sequence $\left\{\kappa_{n}\right\}$ is convergent.

II $^{*}$. The sequence $\left\{\varphi_{n}^{*}\right\}$ is locally uniformly convergent in $\mathbf{D}$.

Note that $\mathrm{I}^{*}$ implies I and II $^{*}$ implies II.

Proposition 3.4. Conditions I and II are equivalent.

Proof. Recall that $\kappa_{n}=\varphi_{n}^{*}\left(\alpha_{n}\right)$. Thus II obviously implies I. On the other hand, I implies that $\left\{\varphi_{n}^{*}(z)\right\}$ cannot diverge locally uniformly to $\infty$, hence II is satisfied, by Proposition 3.2.

Proposition 3.5. Conditions II and III are equivalent.

Proof. First assume that III is satisfied. Then $\left\{\left|\varphi_{n}(z)\right|^{2}\right\}$ tends locally uniformly to zero, hence by $(2.27)\left|\varphi_{n}^{*}(z)\right|^{2}$ is locally uniformly bounded. Next assume that II is satisfied. By Proposition 3.1 there exists a locally uniformly convergent subsequence $\left\{\varphi_{n(s)}^{*}(z)\right\}$ of $\left\{\varphi_{n}^{*}(z)\right\}$. Arguing as in the proof of Proposition 3.2 we see that the whole sequence $\left\{k_{n}(z, z)\right\}$ converges and that $\left\{\varphi_{n}(z)\right\}$ is locally uniformly bounded. Since $\left\{\varphi_{n(s)}^{*}(z)\right\}$ converges it follows by $(2.27)$ that $\left|\zeta_{n(s)}(z)\right|$ converges and the convergence must clearly be locally uniform. It follows, again by (2.27), that the convergence of $\left\{k_{n(s)}(z, z)\right\}$ must be locally uniform, and hence the convergence of $\left\{k_{n}(z, z)\right\}$ is also locally uniform.

Proposition 3.6. Conditions III and IV are equivalent.

Proof. Obviously IV implies III. Assume that III is satisfied. Let $n>p$. We have

$$
\left|k_{n}(z, w)-k_{p}(z, w)\right|=\left|\sum_{m=p+1}^{n} \varphi_{m}(z) \overline{\varphi_{m}(w)}\right|,
$$


hence by Schwartz's inequality

$$
\left|k_{n}(z, w)-k_{p}(z, w)\right| \leq\left(\sum_{m=p+1}^{n}\left|\varphi_{m}(z)\right|^{2}\right)^{1 / 2}\left(\sum_{m=p+1}^{n}\left|\varphi_{m}(w)\right|^{2}\right)^{1 / 2} .
$$

It then follows from III and Cauchy's criterion that $\left\{k_{n}(z, w)\right\}$ converges locally uniformly for $z, w \in \mathbf{D}$.

Corollary 3.7. Assume that condition II is satisfied. Then a subsequence $\left\{\varphi_{n(s)}^{*}\right\}$ of $\left\{\varphi_{n}^{*}\right\}$ is convergent if and only if the subsequence $\left\{\alpha_{n(s)}\right\}$ is convergent.

Proof. This follows from (2.26) and the fact that $\left\{k_{n}(z, w)\right\}$ converges by Propositions 3.5 and 3.6.

Proposition 3.8. Assume that the sequence $\left\{\alpha_{n}\right\}$ converges to a limit $\alpha$. Then conditions I and $\mathrm{I}^{*}$ are equivalent and conditions II and $\mathrm{II}^{*}$ are equivalent.

Proof. That II implies II $^{*}$ follows from Corollary 3.7. That I implies $\mathrm{I}^{*}$ follows from Proposition 3.4. $\left(\varphi_{n}^{*}\left(\alpha_{n}\right)\right.$ converges to $\lim _{n} \varphi_{n}^{*}(\alpha)$ because of the locally uniform convergence.)

We sum up the main results of this section.

Theorem 3.9. A. The conditions I, II, III, and IV are equivalent.

B. If $\left\{\alpha_{n}\right\}$ is convergent, then the conditions I, I*, II, II*, III, and IV are equivalent.

Proof. Follows from Propositions 3.4, 3.5, 3.6, and 3.8.

\section{RECURRENCE RELATIONS}

The sequence $\left\{\varphi_{n}\right\}$ satisfies the following recurrence relation:

$$
\begin{aligned}
\varphi_{n}(z)= & \varepsilon_{n} \frac{z-\alpha_{n-1}}{1-\overline{\alpha_{n}} z} \frac{\kappa_{n}}{\kappa_{n-1}} \varphi_{n-1}(z) \\
& +\delta_{n} \frac{1-\overline{\alpha_{n-1}} z}{1-\overline{\alpha_{n}} z} \frac{\kappa_{n}}{\kappa_{n-1}} \varphi_{n-1}^{*}(z), \quad n=1,2, \ldots, \\
\varphi_{n}^{*}(z)= & -\frac{\overline{\alpha_{n}}}{\left|\alpha_{n}\right|} \bar{\delta}_{n} \frac{z-\alpha_{n-1}}{1-\overline{\alpha_{n}} z} \frac{\kappa_{n}}{\kappa_{n-1}} \varphi_{n-1}(z) \\
& -\frac{\overline{\alpha_{n}}}{\left|\alpha_{n}\right|} \overline{\varepsilon_{n}} \frac{1-\overline{\alpha_{n-1}} z}{1-\overline{\alpha_{n}} z} \frac{\kappa_{n}}{\kappa_{n-1}} \varphi_{n-1}^{*}(z), \quad n=1,2, \ldots
\end{aligned}
$$

The coefficients $\delta_{n}, \varepsilon_{n}$ are given by

$$
\begin{gathered}
\delta_{n}=\frac{\left(1-\alpha_{n-1} \overline{\alpha_{n}}\right) \varphi_{n}\left(\alpha_{n-1}\right)}{\left(1-\left|\alpha_{n-1}\right|^{2}\right) \kappa_{n}}, \\
\varepsilon_{n}=-\frac{\overline{\alpha_{n}}}{\left|\alpha_{n}\right|} \frac{\left(1-\overline{\alpha_{n-1}} \alpha_{n}\right) \overline{\varphi_{n}^{*}\left(\alpha_{n-1}\right)}}{\left(1-\left|\alpha_{n-1}\right|^{2}\right) \kappa_{n}} .
\end{gathered}
$$

It follows from (3.1) with $z=\alpha_{n-1}$ that $\left|\varepsilon_{n}\right| \neq 0$. 
Note that

$$
\frac{\delta_{n}}{\varepsilon_{n}}=-\frac{\left|\alpha_{n}\right|}{\overline{\alpha_{n}}} \frac{\left(1-\alpha_{n-1} \overline{\alpha_{n}}\right) \varphi_{n}\left(\alpha_{n-1}\right)}{\left(1-\overline{\alpha_{n-1}} \alpha_{n}\right) \overline{\varphi_{n}^{*}\left(\alpha_{n-1}\right)}} .
$$

By substituting for $\varphi_{n-1}^{*}(z)$ from (4.2) into (4.1) we get the three-term recurrence formula

$$
\begin{array}{r}
\varphi_{n}(z)=-\frac{\alpha_{n}}{\left|\alpha_{n}\right|} \frac{\delta_{n}}{\overline{\varepsilon_{n}}} \varphi_{n}^{*}(z)+\frac{1}{\overline{\varepsilon_{n}}}\left[\left|\varepsilon_{n}\right|^{2}-\left|\delta_{n}\right|^{2}\right] \frac{z-\alpha_{n-1}}{1-\overline{\alpha_{n}} z} \frac{\kappa_{n}}{\kappa_{n-1}} \varphi_{n-1}(z), \\
n=1,2, \ldots .
\end{array}
$$

Furthermore, the sequence $\left\{\psi_{n}\right\}$ of associated functions (cf. (2.22)-(2.23)) satisfies the relations

$$
\begin{gathered}
\psi_{n}(z)=\varepsilon_{n} \frac{z-\alpha_{n-1}}{1-\overline{\alpha_{n}} z} \frac{\kappa_{n}}{\kappa_{n-1}} \psi_{n-1}(z)-\delta_{n} \frac{1-\overline{\alpha_{n-1}} z}{1-\overline{\alpha_{n}} z} \frac{\kappa_{n}}{k_{n-1}} \psi_{n-1}^{*}(z), \\
n=1,2, \ldots, \\
\begin{array}{c}
\psi_{n}^{*}(z)=\frac{\overline{\alpha_{n}}}{\left|\alpha_{n}\right|} \bar{\delta}_{n} \frac{z-\alpha_{n-1}}{1-\overline{\alpha_{n}} z} \frac{\kappa_{n}}{\kappa_{n-1}} \psi_{n-1}(z)-\frac{\overline{\alpha_{n}}}{\left|\alpha_{n}\right|} \overline{\frac{1-\overline{\varepsilon_{n}}}{\left(-\overline{\alpha_{n-1}} z\right.}} \frac{\kappa_{n}}{1-\overline{\alpha_{n}} z} \psi_{n-1}^{*}(z), \\
n=1,2, \ldots
\end{array} \\
\psi_{n}(z)=\frac{\alpha_{n}}{\left|\alpha_{n}\right|} \frac{\delta_{n}}{\overline{\varepsilon_{n}}} \psi_{n}^{*}(z)+\frac{1}{\overline{\varepsilon_{n}}}\left[\left|\varepsilon_{n}\right|^{2}-\left|\delta_{n}\right|^{2}\right] \frac{z-\alpha_{n-1}}{1-\overline{\alpha_{n}} z} \frac{\kappa_{n}}{\kappa_{n-1}} \psi_{n-1}(z), \\
n=1,2, \ldots
\end{gathered}
$$

In particular, it follows from (4.2), (4.7), (4.9), (4.10) that the functions $-\psi_{n}^{*}(z) / \varphi_{n}^{*}(z)$ are the even approximants and the functions $\psi_{n}(z) / \varphi_{n}(z)$ are the odd approximants of a continued fraction of a certain form. (Continued fractions of this kind are sometimes called Nevanlinna-Pick fractions.) For proofs and more details concerning the recurrence relations presented here, see $[4,6,12]$.

Some auxiliary technical results used in this section and in $\S \S 5-6$ are discussed in $\S 7$.

We shall consider some properties connected with the recurrence coefficients $\left\{\delta_{n}\right\},\left\{\varepsilon_{n}\right\}$. We recall that convergence of an infinite product means convergence to a finite number different from zero.

V. The sequence $\left\{\prod_{m=1}^{n}\left[\left|\varepsilon_{m}\right|^{2}-\left|\delta_{m}\right|^{2}\right]\right\}$ is bounded away form zero.

$\mathrm{V}^{*}$. The product $\prod_{m=1}^{\infty}\left[\left|\varepsilon_{m}\right|^{2}-\left|\delta_{m}\right|^{2}\right]$ converges.

$\mathrm{V}^{* *}$. The series $\sum_{m=1}^{\infty}\left|\delta_{m}\right|^{2}$ converges.

Proposition 4.1. Conditions $\mathrm{I}$ and $\mathrm{V}$ are equivalent.

Proof. The result follows from Corollary 7.5.

Proposition 4.2. Assume that the sequence $\left\{\alpha_{n}\right\}$ converges to a limit $\alpha$. Then conditions $\mathrm{I}^{*}$ and $\mathrm{V}^{*}$ are equivalent.

Proof. Follows from (7.23). 
Proposition 4.3. Assume that $\alpha_{n}=\alpha$ for all $n$. Then conditions $\mathrm{V}^{*}$ and $\mathrm{V}^{* *}$ are equivalent.

Proof. It follows from (4.5) that in this situation $\left|\varepsilon_{n}\right|=1$. It is well known that the product $\prod_{m=1}^{\infty}\left[1-\left|\delta_{m}\right|^{2}\right]$ converges if and only if the series $\sum_{m=1}^{\infty}\left|\delta_{m}\right|^{2}$ converges.

We sum up our main results so far.

Theorem 4.4. A. The conditions I, II, III, IV, V are equivalent.

B. If $\left\{\alpha_{n}\right\}$ is convergent, then the conditions under $\mathrm{A}$ are also equivalent to the conditions $\mathrm{I}^{*}, \mathrm{II}^{*}, \mathrm{~V}^{*}$.

C. If $\alpha_{n}=\alpha$ for all $n$, then the conditions under $\mathrm{A}$ and $\mathrm{B}$ are also equivalent to the condition $\mathrm{V}^{* *}$.

Proof. Follows from Theorem 3.9 and Propositions 4.1, 4.2, and 4.3.

\section{SZEGÖ CONDITION}

We shall in this section consider relationships between the Szegö condition (see VII below), cf. (1.4), completeness of the systems $\left\{\varphi_{n}\left(e^{i \theta}\right)\right\}$ in $L_{2}(\mu)$, and the conditions discussed in $\S \S 3-4$.

Proposition 5.1. The closed linear span of $\left\{B_{n}\left(e^{i \theta}\right): n=0,1,2, \ldots\right\}$ in $L_{2}(\mu)$ equals the closed linear span of $\left\{e^{i n \theta}: n=0,1,2, \ldots\right\}$ in $L_{2}(\mu)$ if $\sum_{m=1}^{\infty}\left(1-\left|\alpha_{m}\right|\right)=\infty$.

Proof. See [4, p. 63].

We now introduce the following two conditions.

VI. The sequence $\left\{\varphi_{n}\left(e^{i \theta}\right)\right\}$ is not complete in $L_{2}(\mu)$.

VII. (Szegö condition) $\int_{-\pi}^{\pi} \ln \mu^{\prime}(\theta) d \theta>-\infty$.

Proposition 5.2. The conditions VI and VII are equivalent.

Proof. Note that $\left\{\varphi_{n}\right\}$ being complete is equivalent to $\left\{B_{n}\right\}$ being complete. Recall that we have assumed $\left|\alpha_{n}\right| \leq R<1$ for all $n$. It follows from Proposition 5.1 that $\left\{\varphi_{n}\left(e^{i \theta}\right)\right\}$ is complete in $L_{2}(\mu)$ if and only if $\left\{e^{i n \theta}: n=0,1,2\right.$, $\ldots\}$ is complete in $L_{2}(\mu)$. It is a known result that $\left\{e^{i n \theta}: n=0,1,2, \ldots\right\}$ is not complete in $L_{2}(\mu)$ if and only if condition VII is satisfied. See, e.g., [19, 22].

We introduce the notation $P(z, \alpha)$ and $p_{n}(\theta)$ for

$$
\begin{aligned}
& P(z, \alpha)=\frac{1-|\alpha|^{2}}{|z-\alpha|^{2}}, \\
& p_{n}(\theta)=P\left(e^{i \theta}, \alpha_{n}\right) .
\end{aligned}
$$

Note that $P\left(e^{i \theta}, \alpha\right)$ is the Poisson kernel, with

$$
\int_{-\pi}^{\pi} P\left(e^{i \theta}, \alpha\right) d \theta=2 \pi \text {. }
$$

Recall that some auxiliary technical results used in this and other sections are discussed in $\S 7$. 
Proposition 5.3. Condition VII implies condition I.

Proof. The functions $p_{n}(\theta)$ are uniformly bounded an uniformly bounded away from zero for $\theta \in[-\pi, \pi]$ and all $n$. Let $m$ and $M$ be such that

$$
0<m \leq p_{n}(\theta) \leq M<\infty .
$$

Then it can be shown by elementary calculations that

$$
\int_{-\pi}^{\pi} p_{n}(\theta) \ln \left[\frac{\mu^{\prime}(\theta)}{p_{n}(\theta)}\right] d \theta>M \int_{-\pi}^{\pi} \ln \mu^{\prime}(\theta) d \theta-\left[(M-m) \frac{1}{k_{0}^{2}}+2 \pi \ln M\right]
$$

and

$$
\int_{-\pi}^{\pi} p_{n}(\theta) \ln \left[\frac{\mu^{\prime}(\theta)}{p_{n}(\theta)}\right] d \theta \leq m \int_{-\pi}^{\pi} \ln \mu^{\prime}(\theta) d \theta-\left[(M-m) \frac{1}{k_{0}^{2}}+2 \pi \ln m\right] .
$$

It follows by Proposition 7.6 that if $\int_{-\pi}^{\pi} \ln \mu^{\prime}(\theta) d \theta>-\infty$, then $\left\{\kappa_{n}^{2}\right\}$ is bounded.

We shall in the next section, by invoking deeper function theoretic results, prove the converse of this result. Here we shall indicate by more elementary means how I implies VI and hence VII, in the special situation that $\alpha_{n}=\alpha$ for all $n$.

Proposition 5.4. Assume that $\alpha_{n}=\alpha$ for all $n$. Then condition I implies condition VI.

Proof. We define

$$
F(z)=\frac{1-\bar{\alpha} z}{z-\alpha} .
$$

Denote by $\beta_{n}$ the $n$th Fourier coefficient of $F$ with respect to the system $\left\{\varphi_{n}\right\}$, i.e.,

$$
\beta_{n}=\left\langle F, \varphi_{n}\right\rangle .
$$

Then by (7.37) $\beta_{n-1}=\frac{\bar{\alpha}}{\mid \alpha} \frac{\bar{\delta}_{n}}{\kappa_{N-1}}$. We recall that in this situation $\left|\varepsilon_{n}\right|=1$ for all $n$ (cf. (4.5)). It follows from (7.41) that

$$
\left(1-|\alpha|^{2}\right) \sum_{m=1}^{n}\left|\beta_{n-1}\right|^{2}=\left(1-|\alpha|^{2}\right)\left(\frac{1}{\kappa_{0}^{2}}-\frac{1}{\kappa_{n}^{2}}\right) .
$$

(Note that we may in this argument set $\alpha_{0}=\alpha$ since formulas (4.1), (4.2), (4.4), (4.5) are valid for $n=1$ with an arbitrary value of $\alpha_{0}$.) It follows that if $\left\{\kappa_{n}\right\}$ is bounded, then

$$
\sum_{m=1}^{\infty}\left|\beta_{m-1}\right|^{2}<\frac{1}{\kappa_{0}^{2}}
$$

On the other hand

$$
\langle F, F\rangle=\int_{-\pi}^{\pi}\left|\frac{1-\bar{\alpha} e^{i \theta}}{e^{i \theta}-\alpha}\right|^{2} d \mu(\theta)=\int_{-\pi}^{\pi} d \mu(\theta)=\frac{1}{\kappa_{0}^{2}} .
$$

Thus if $\left\{\kappa_{n}\right\}$ is bounded, Parseval's equality is not valid for the function $F \in$ $L_{2}(\mu)$, and so $\left\{\varphi_{n}\right\}$ is not complete in $L_{2}(\mu)$. 


\section{LIMIT FUNCTIONS}

The discussion in $\S 3$ shows that $\left\{\varphi_{n}^{*}(z)\right\}$ may exhibit two different kinds of convergence behavior in $\mathbf{D}:(1)\left\{\varphi_{n}^{*}(z)\right\}$ diverges locally uniformly to $\infty$. (2) For each accumulation point $\alpha$ of $\left\{\alpha_{n}\right\}$ there exists an analytic function $\pi_{\alpha}(z)$ without zeros such that $\left\{\varphi_{n(s)}^{*}(z)\right\}$ converges locally uniformly to $\pi_{\alpha}(z)$ if $\left\{\alpha_{n(s)}\right\}$ converges to $\alpha$. We shall sometimes use the notation $\frac{1}{\pi_{\alpha}(z)}$ also in case (1), and then mean the zero function. In Theorem 6.12 we shall give explicit representations of the functions $\pi_{\alpha}(z)$.

We shall first (in Proposition 6.5) show that the functions $\frac{1}{\pi_{\alpha}(z)}$ belong to the Hardy class $\mathrm{H}_{2}$. (For the definition of this space and other basic material for this section, see $[18,25,36,37]$.) We shall make use of the fact that the integral $\int_{-\pi}^{\pi} \frac{p_{n}(\theta) d \theta}{\left|\varphi_{n}^{*}\left(e^{i \theta}\right)\right|^{2}}$ is independent of $n$ (Lemma 6.3). To obtain this we utilize the interpolation property $(2.28)$ of $\psi_{n}^{*}(z) / \varphi_{n}^{*}(z)$ to $\Omega_{\mu}(z)$ at the origin.

We again recall that some auxiliary technical results used in this and other sections are discussed in $\S 7$.

Lemma 6.1. The following equality holds:

$$
\operatorname{Re}\left[\frac{\psi_{n}^{*}\left(e^{i \theta}\right)}{\varphi_{n}^{*}\left(e^{i \theta}\right)}\right]=\frac{p_{n}(\theta)}{\left|\varphi_{n}^{*}\left(e^{i \theta}\right)\right|^{2}} .
$$

Proof. The real part of $\psi_{n}^{*}\left(e^{i \theta}\right) / \varphi_{n}^{*}\left(e^{i \theta}\right)$ may be written

$$
\operatorname{Re}\left[\frac{\psi_{n}^{*}\left(e^{i \theta}\right)}{\varphi_{n}^{*}\left(e^{i \theta}\right)}\right]=\frac{\psi_{n}^{*}\left(e^{i \theta}\right) \overline{\varphi_{n}^{*}\left(e^{i \theta}\right)}+\overline{\psi_{n}^{*}\left(e^{i \theta}\right)} \varphi_{n}^{*}\left(e^{i \theta}\right)}{2\left|\varphi_{n}^{*}\left(e^{i \theta}\right)\right|^{2}} .
$$

It is readily verified that

$$
\overline{\varphi_{n}^{*}\left(e^{i \theta}\right)}=\frac{1}{B_{n}\left(e^{i \theta}\right)} \varphi_{n}\left(e^{i \theta}\right), \quad \overline{\psi_{n}^{*}\left(e^{i \theta}\right)}=\frac{1}{B_{n}\left(e^{i \theta}\right)} \psi_{n}\left(e^{i \theta}\right) .
$$

By combining (7.1) and (7.23) we get

$$
\varphi_{n}^{*}(z) \psi_{n}(z)+\varphi_{n}(z) \psi_{n}^{*}(z)=\frac{2 z B_{n}(z)\left(1-\left|\alpha_{n}\right|^{2}\right)}{\left(z-\alpha_{n}\right)\left(1-\overline{\alpha_{n}} z\right)} .
$$

Formula (6.1) then follows from (5.1)-(5.2) and (6.2)-(6.4).

Lemma 6.2. For $z \in \mathbf{D}$ the following inequality holds:

$$
\operatorname{Re}\left[\frac{\psi_{n}^{*}(z)}{\varphi_{n}^{*}(z)}\right] \geq \frac{1-\left|\alpha_{n}\right|^{2}}{\left|1-\overline{\alpha_{n}} z\right|^{2}\left|\varphi_{n}^{*}(z)\right|^{2}} .
$$

Proof. The function $\operatorname{Re}\left[\psi_{n}^{*}(z) / \varphi_{n}^{*}(z)\right]$ is harmonic in $\mathbf{D} \cup \mathbf{T}$, therefore by Poisson's formula we may write (since (6.1) holds):

$$
\operatorname{Re}\left[\frac{\psi_{n}^{*}(z)}{\varphi_{n}^{*}(z)}\right]=\frac{1}{2 \pi} \int_{-\pi}^{\pi} P\left(e^{i \theta}, z\right) \frac{p_{n}(\theta) d \theta}{\left|\varphi_{n}^{*}\left(e^{i \theta}\right)\right|^{2}}
$$

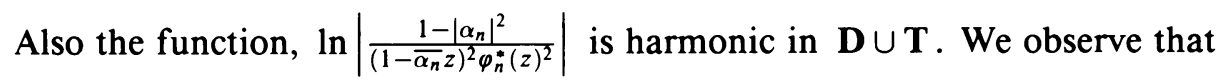

$$
\frac{1-|\alpha|^{2}}{\left|1-\bar{\alpha} e^{i \theta}\right|^{2}}=P\left(e^{i \theta}, \alpha\right)
$$


for arbitrary $\alpha \in \mathbf{D}$. Hence (again by the Poisson formula)

$$
\ln \left|\frac{1-\left|\alpha_{n}\right|^{2}}{\left(1-\overline{\alpha_{n}} z\right)^{2} \varphi_{n}^{*}(z)^{2}}\right|=\frac{1}{2 \pi} \int_{-\pi}^{\pi} P\left(e^{i \theta}, z\right) \ln \left[\frac{p_{n}(\theta)}{\left|\varphi_{n}^{*}\left(e^{i \theta}\right)\right|^{2}}\right] d \theta .
$$

From the inequality between weighted geometric and arithmetic means (cf. (7.24)) we obtain

$$
\frac{1}{2 \pi} \int_{-\pi}^{\pi} P\left(e^{i \theta}, z\right) \frac{p_{n}(\theta)}{\left|\varphi_{n}^{*}\left(e^{i \theta}\right)\right|^{2}} d \theta \geq \exp \left[\frac{1}{2 \pi} \int_{-\pi}^{\pi} P\left(e^{i \theta}, z\right) \ln \left[\frac{p_{n}(\theta)}{\left|\varphi_{n}^{*}\left(e^{i \theta}\right)\right|^{2}}\right] d \theta\right] .
$$

Substitution from (6.6) and (6.8) then yields (6.5).

Lemma 6.3. The following equality holds:

$$
\int_{-\pi}^{\pi} \frac{p_{n}(\theta) d \theta}{\left|\varphi_{n}^{*}\left(e^{i \theta}\right)\right|^{2}}=\frac{2 \pi}{\kappa_{0}^{2}}, \quad n=0,1,2, \ldots
$$

Proof. It follows from (2.28) that

$$
\frac{\psi_{n}^{*}(0)}{\varphi_{n}^{*}(0)}=\Omega_{\mu}(0)=\frac{1}{\kappa_{0}^{2}}
$$

The function $\psi_{n}^{*}(z) / \varphi_{n}^{*}(z)$ then has a Taylor series expansion

$$
\frac{\psi_{n}^{*}(z)}{\varphi_{n}^{*}(z)}=\frac{1}{\kappa_{0}^{2}}+\sum_{m=1}^{\infty} c_{m}^{(n)} z^{m}
$$

valid in a neighborhood of $\mathbf{D} \cup \mathbf{T}$, since $\varphi_{n}^{*}(z)$ has no zeros in $\mathbf{D} \cup \mathbf{T}$. From this follows that $\operatorname{Re}\left[\psi_{n}^{*}\left(e^{i \theta}\right) / \varphi_{n}^{*}\left(e^{i \theta}\right)\right]$ has the following series expansion:

$$
\operatorname{Re}\left[\psi_{n}^{*}\left(e^{i \theta}\right) / \varphi_{n}^{*}\left(e^{i \theta}\right)\right]=\frac{1}{\kappa_{0}^{2}}+\frac{1}{2} \sum_{m=1}^{\infty}\left[c_{m}^{(n)} e^{i m \theta}+c_{m}^{(n)} e^{-i m \theta}\right]
$$

uniformly convergent for $\theta \in[-\pi, \pi]$.

Term by term integration of the expansion (6.13) leads to

$$
\int_{-\pi}^{\pi} \operatorname{Re}\left[\frac{\psi_{n}^{*}\left(e^{i \theta}\right)}{\varphi_{n}^{*}\left(e^{i \theta}\right)}\right] d \theta=\frac{2 \pi}{\kappa_{0}^{2}}
$$

Formula (6.10) now follows from Lemma 6.1.

Lemma 6.4. There exists a constant $B$ such that

$$
\int_{-\pi}^{\pi} \frac{d \theta}{\left|\varphi_{n}^{*}\left(r e^{i \theta}\right)\right|^{2}} \leq B
$$

for all $n$ and all $r \in[0,1]$.

Proof. Because of (5.4) Lemma 6.3 implies that there exists a constant $B$ such that

$$
\int_{-\pi}^{\pi} \frac{d \theta}{\left|\varphi_{n}^{*}\left(e^{i \theta}\right)\right|^{2}} \leq B
$$

for all $n$. The integral

$$
\int_{-\pi}^{\pi} \frac{d \theta}{\left|\varphi_{n}^{*}\left(r e^{i \theta}\right)\right|^{2}}
$$


is a nondecreasing function of $r$ for $r \in[0,1]$ (see, e.g., [36, 37]), and so the inequality (6.15) follows from (6.16).

Proposition 6.5. Every function $\frac{1}{\pi_{\alpha}(z)}$ belongs to the Hardy space $H_{2}$, i.e., there exists a constant $B$ such that

$$
\int_{-\pi}^{\pi} \frac{1}{\left|\pi_{\alpha}\left(r e^{i \theta}\right)\right|^{2}} d \theta \leq B
$$

for all $r \in[0,1)$.

Proof. Let $\alpha_{n(s)} \underset{s \rightarrow \infty}{\longrightarrow} \alpha$. For fixed $r \in[0,1), 1 /\left|\varphi_{n(s)}^{*}(z)\right|^{2}$ converges uniformly to $1 /\left|\pi_{\alpha}(z)\right|^{2}$ for $|z| \leq r$. It follows that

$$
\int_{-\pi}^{\pi} \frac{d \theta}{\left|\varphi_{n(s)}^{*}\left(r e^{i \theta}\right)\right|^{2}}
$$

converges to

$$
\int_{-\pi}^{\pi} \frac{d \theta}{\left|\pi_{\alpha}\left(r e^{i \theta}\right)\right|^{2}}
$$

and so (6.17) follows from Lemma 6.4.

Since $1 / \pi_{\alpha}(z)$ belongs to $H_{2}$, the limit of $1 / \pi_{\alpha}\left(r e^{i \theta}\right)$, hence of $\pi_{\alpha}\left(r e^{i \theta}\right)$, exists a.e. on $[-\pi, \pi]$ as $r$ tends to 1 . We define $\pi_{\alpha}\left(e^{i \theta}\right)$ by

$$
\pi_{\alpha}\left(e^{i \theta}\right)=\lim _{r \rightarrow 1^{-}} \pi_{\alpha}\left(r e^{i \theta}\right) .
$$

Proposition 6.6. For $z \in \mathbf{D}$ the following inequality holds:

$$
\frac{1-|\alpha|^{2}}{|1-\bar{\alpha} z|^{2}\left|\pi_{\alpha}(z)\right|^{2}} \leq \operatorname{Re} \Omega_{\mu}(z) \text {. }
$$

Proof. This follows from Lemma 6.2, the convergence of a subsequence of $\left\{\varphi_{n}^{*}(z)\right\}$ to $\pi_{\alpha}(z)$ and the convergence of $\left\{\psi_{n}^{*}(z) / \varphi_{n}^{*}(z)\right\}$ to $\Omega_{\mu}(z)$ according to $(2.29)$ (recall $(2.30)$ ).

It is known that $\operatorname{Re} \Omega_{\mu}(z)=\int_{-\pi}^{\pi} P\left(z, e^{i \theta}\right) d \mu(\theta)$ has radial limit equal to $2 \pi \mu^{\prime}(\theta)$ a.e., i.e.

$$
\lim _{r \rightarrow 1^{-}} \operatorname{Re} \Omega_{\mu}\left(r e^{i \theta}\right)=2 \pi \mu^{\prime}(\theta) \quad \text { a.e. }
$$

(see e.g. [36]). It then follows from Proposition 6.6 (cf. also (6.7)) that

$$
\frac{P\left(e^{i \theta}, \alpha\right)}{\left|\pi_{\alpha}\left(e^{i \theta}\right)\right|^{2}} \leq 2 \pi \mu^{\prime}(\theta) \quad \text { a.e. }
$$

Proposition 6.7. The following inequality holds:

$$
\pi_{\alpha}(\alpha)^{2} \geq \frac{1}{2 \pi} \exp \left(-\frac{1}{2 \pi} \int_{-\infty}^{\pi} P\left(e^{i \theta}, \alpha\right) \ln \left[\frac{\mu^{\prime}(\theta)}{P\left(e^{i \theta}, \alpha\right)}\right] d \theta\right) .
$$

Proof. It follows from (6.21) that

$$
\frac{1}{2 \pi} \int_{-\pi}^{\pi} P\left(e^{i \theta}, \alpha\right) \ln \left[\frac{\mu^{\prime}(\theta)}{P\left(e^{i \theta}, \alpha\right)}\right] d \theta \geq \frac{1}{2 \pi} \int_{-\pi}^{\pi} P\left(e^{i \theta}, \alpha\right) \ln \frac{1}{2 \pi\left|\pi_{\alpha}\left(e^{i \theta}\right)\right|^{2}} d \theta .
$$


Poisson's formula gives

$$
\frac{1}{2 \pi} \int_{-\pi}^{\pi} P\left(e^{i \theta}, \alpha\right) \ln \frac{1}{2 \pi\left|\pi_{\alpha}\left(e^{i \theta}\right)\right|^{2}} d \theta=\ln \frac{1}{2 \pi\left|\pi_{\alpha}(\alpha)\right|^{2}} .
$$

Combining (6.23) and (6.24), taking into account that $\pi_{\alpha}(\alpha)$ is real since $\varphi_{n}^{*}\left(\alpha_{n}\right)$ is real, we get

$$
\frac{1}{2 \pi} \int_{-\pi}^{\pi} P\left(e^{i \theta}, \alpha\right) \ln \left[\frac{\mu^{\prime}(\theta)}{P\left(e^{i \theta}, \alpha\right)}\right] d \theta \geq \ln \frac{1}{2 \pi \pi_{\alpha}(\alpha)^{2}},
$$

which is equivalent to $(6.22)$.

Proposition 6.8. Assume that $\alpha_{n(s)} \underset{s \rightarrow \infty}{\longrightarrow} \alpha$. Then

$$
\lim _{s \rightarrow \infty} \kappa_{n(s)}^{2}=\frac{1}{2 \pi} \exp \left[-\frac{1}{2 \pi} \int_{-\pi}^{\pi} P\left(e^{i \theta}, \alpha\right) \ln \left[\frac{\mu^{\prime}(\theta)}{P\left(e^{i \theta}, \alpha\right)}\right] d \theta\right] .
$$

Proof. Follows from Proposition 7.6 and Proposition 6.7. (Recall that $\kappa_{n}=$ $\varphi_{n}^{*}\left(\alpha_{n}\right)$.)

Proposition 6.9. Conditions I and VII are equivalent.

Proof. This follows from the proof of Proposition 5.3 and Proposition 6.8. (Recall (5.4) and Corollary 3.3.)

We sum up our main result on equivalences here.

Theorem 6.10. A. Conditions I, II, III, IV, V, VI, VII are equivalent.

B. Assume that $\left\{\alpha_{n}\right\}$ converges to a limit $\alpha$. Then conditions $\mathrm{I}^{*}, \mathrm{II}^{*}, \mathrm{~V}^{*}$ are equivalent to the conditions under $\mathrm{A}$.

C. Assume that all $\alpha_{n}$ equals a fixed value $\alpha$. Then condition $\mathrm{V}^{* *}$ is equivalent to the conditions under $\mathrm{A}$ and $\mathrm{B}$.

Proof. Follows from Theorem 4.4, Proposition 5.2, and Proposition 6.9.

Proposition 6.11. If condition VII is satisfied, the following equality holds:

$$
\frac{1}{\left|\pi_{\alpha}\left(e^{i \theta}\right)\right|^{2}}=2 \pi \frac{\mu^{\prime}(\theta)}{P\left(e^{i \theta}, \alpha\right)} \text { a.e. }
$$

Proof. We recall that (6.21) is valid. Assume that strict inequality holds on a set of positive measure. It follows from the proof of Proposition 6.7 that then there would also be strict inequality in (6.22) in case the integral is finite. This contradicts Proposition 5.3.

Let $H(z)$ be an arbitrary function in $H_{2}$. Such a function has a representation of the form

$$
\begin{aligned}
H(z)= & e^{i \lambda} z^{\nu} \prod_{m=1}^{\infty} \zeta\left(z_{m}, z\right) \exp \left[\frac{1}{2 \pi} \int_{-\pi}^{\pi} D\left(e^{i \theta}, z\right) d \omega(\theta)\right] \\
& \cdot \exp \left[\frac{1}{2 \pi} \int_{-\pi}^{\pi} D\left(e^{i \theta}, z\right) \ln \left|H\left(e^{i \theta}\right)\right| d \theta\right]
\end{aligned}
$$

where $\lambda \in \mathbf{R}, \nu \in \mathbf{N}_{0}, z_{1}, \ldots, z_{q}, \ldots \in \mathbf{D}$, and $\omega$ is a singular (nonnegative) measure. (See e.g. $[18,36]$.) 
Theorem 6.12. Assume that $\left\{\alpha_{n(s)}\right\}$ converges to $\alpha$. Then $\pi_{\alpha}(z)=\lim _{s \rightarrow \infty} \varphi_{n(s)}^{*}(z)$ has the following representation:

$$
\pi_{\alpha}(z)=\frac{e^{-i \lambda}}{\sqrt{2 \pi}} \frac{\sqrt{1-|\alpha|^{2}}}{1-\bar{\alpha} z} \exp \left[-\frac{1}{4 \pi} \int_{-\pi}^{\pi} D\left(e^{i \theta}, z\right) \ln \mu^{\prime}(\theta) d \theta\right]
$$

where $\lambda \in \mathbf{R}$.

Proof. We recall that the function $1 / \pi_{\alpha}(z)$ belongs to $H_{2}$ and has no zeros in D. We note that

$$
\frac{1}{4 \pi} \int_{-\pi}^{\pi} D\left(e^{i \theta}, z\right) \ln 2 \pi d \theta=\frac{1}{2} \ln (2 \pi) .
$$

It follows from (6.27), (6.28), and (6.30) that we may write

$$
\begin{aligned}
\frac{1}{\pi_{\alpha}(z)}= & \sqrt{2 \pi} e^{i \lambda} \exp \left[\frac{1}{2 \pi} \int_{-\pi}^{\pi} D\left(e^{i \theta}, z\right) d \omega(\theta)\right] \\
& \cdot \exp \left[\frac{1}{4 \pi} \int_{-\pi}^{\pi} D\left(\left(e^{i \theta}, z\right) \ln \left[\frac{\mu^{\prime}(\theta)}{P\left(e^{i \theta}, \alpha\right)}\right] d \theta\right]\right.
\end{aligned}
$$

where $\lambda \in \mathbf{R}$, and hence

$$
\begin{aligned}
\frac{1}{\left|\pi_{\alpha}(z)\right|}= & \sqrt{2 \pi} \exp \left[\frac{1}{2 \pi} \int_{-\pi}^{\pi} P\left(e^{i \theta}, z\right) d \omega(\theta)\right] \\
& \cdot \exp \left[\frac{1}{4 \pi} \int_{-\pi}^{\pi} P\left(\left(e^{i \theta}, z\right) \ln \left[\frac{\mu^{\prime}(\theta)}{P\left(e^{i \theta}, \alpha\right)}\right] d \theta\right]\right.
\end{aligned}
$$

From Proposition 6.8 then follows

$$
\frac{1}{\pi_{\alpha}(\alpha)}=\exp \left[\frac{1}{2 \pi} \int_{-\pi}^{\pi} P\left(e^{i \theta}, \alpha\right) d \omega(\theta)\right] \cdot \frac{1}{\pi_{\alpha}(\alpha)} .
$$

Consequently $\int_{-\pi}^{\pi} P\left(e^{i \theta}, \alpha\right) d \omega(\theta)=0$, hence $\omega \equiv 0$. The function $\frac{\sqrt{1-|\alpha|^{2}}}{1-\bar{\alpha} z}$ belongs to $\mathrm{H}_{2}$ and can hence by (6.7) and (6.28) be written as

$$
\frac{\sqrt{1-|\alpha|^{2}}}{1-\bar{\alpha} z}=\exp \left[\frac{1}{4 \pi} \int_{-\pi}^{\pi} D\left(e^{i \theta}, z\right) \ln P\left(e^{i \theta}, \alpha\right) d \theta\right] \text {. }
$$

By combining (6.31) and (6.34), taking into account that $\omega \equiv 0$, we obtain

$$
\frac{1}{\pi_{\alpha}(z)}=e^{i \lambda} \exp \left[\frac{1}{4 \pi} \int_{-\pi}^{\pi} D\left(e^{i \theta}, z\right) \ln \mu^{\prime}(\theta) d \theta\right] \frac{(1-\bar{\alpha} z)}{\sqrt{1-|\alpha|^{2}}}
$$

which is equivalent to (6.29).

Note that $\pi_{\alpha}(\alpha)$ is a real number. The value of $\lambda$ is therefore the imaginary part of $-\frac{1}{4 \pi} \int_{\pi}^{\pi} D\left(e^{i \theta}, \alpha\right) \ln \mu^{\prime}(\theta) d \theta$. We recall (cf. (1.3)) that the spectral factor $\sigma_{\mu}$ of $\mu$ is defined by

$$
\sigma_{\mu}(z)=\sqrt{2 \pi} \exp \left[\frac{1}{4 \pi} \int_{-\pi}^{\pi} D\left(e^{i \theta}, z\right) \ln \mu^{\prime}(\theta) d \theta\right] .
$$

Strictly speaking the definition is for the situation that the Szegö condition (VII) is satisfied, but $\sigma_{\mu}(z)$ may be taken to mean zero otherwise. 
We may also introduce the modified spectral factor $\sigma_{\mu}(\alpha, z)$ defined by

$$
\sigma_{\mu}(\alpha, z)=\frac{1-\bar{\alpha} z}{\sqrt{1-|\alpha|^{2}}} \sigma_{\mu}(z)
$$

Corollary 6.13. The function $\pi_{\alpha}(z)$ may be written as

$$
\pi_{\alpha}(z)=e^{-i \lambda} \frac{1}{\sigma_{\mu}(\alpha, z)}, \quad \lambda \in \mathbf{R} .
$$

Proof. Follows immediately from (6.36), (6.37), and Theorem 6.12.

Theorem 6.14. Let $k_{n}(z, w)$ denote the reproducing kernels associated with $\mathscr{L}_{n}$ and $\mu$, as in (2.24). Assume that the equivalent conditions I-VII are satisfied. Then

$$
\lim _{n \rightarrow \infty} k_{n}(z, w)=\frac{1}{\sigma_{\mu}(z) \overline{\sigma_{\mu}(w)}(1-z \bar{w})},
$$

locally uniformly for $z, w \in \mathbf{D}$.

Proof. The locally uniform convergence is included in the condition IV. Let $\alpha$ be an accumulation point for $\left\{\alpha_{n}\right\}$ with $\alpha_{n(s)} \underset{s \rightarrow \infty}{\longrightarrow} \alpha$. Since $\left\{\varphi_{n}(z)\right\}$ tends to zero, it follows from (2.26) that

$$
\lim _{s \rightarrow \infty} k_{n(s)}(z, w)=\frac{\pi_{\alpha}(z) \overline{\pi_{\alpha}(w)}}{1-\zeta(\alpha, z) \overline{\zeta(\alpha, w)}} .
$$

Taking into account (6.37)-(6.38) we find by direct calculation that

$$
\frac{\pi_{\alpha}(z) \overline{\pi_{\alpha}(w)}}{1-\zeta(\alpha, z) \overline{\zeta(\alpha, w)}}=\frac{1}{\sigma_{\mu}(z) \overline{\sigma_{\mu}(w)}(1-z \bar{w})},
$$

from which the result follows.

\section{AUXILIARY TECHNICAL RESUltS}

We shall in this section prove some of the more technical results that are used in the proofs of the main results in $\S$ \$-6.

Proposition 7.1. The following formula is valid:

$$
\begin{array}{r}
\varphi_{n}^{*}(z) \psi_{n}(z)+\varphi_{n}(z) \psi_{n}^{*}(z)=\frac{\kappa_{n}^{2}}{\kappa_{0}^{2}} \prod_{m=1}^{n}\left[\left|\varepsilon_{m}\right|^{2}-\left|\delta_{m}\right|^{2}\right] \frac{2 z B_{n}(z)}{\left(z-\alpha_{n}\right)\left(1-\overline{\alpha_{n}} z\right)}, \\
n=1,2, \ldots .
\end{array}
$$

Proof. By using (4.1), (4.2), (4.8), (4.9) we get by direct calculation

$$
\begin{aligned}
\varphi_{n}^{*}(z) & \psi_{n}(z)+\varphi_{n}(z) \psi_{n}^{*}(z) \\
= & \frac{\kappa_{n}^{2}}{\kappa_{n-1}^{2}} \frac{\overline{\alpha_{n}}}{\left|\alpha_{n}\right|} \frac{\left(\alpha_{n-1}-z\right)\left(1-\overline{\alpha_{n-1}} z\right)}{\left(1-\overline{\alpha_{n}} z\right)^{2}} \\
& \cdot\left[\left|\varepsilon_{n}\right|^{2}-\left|\delta_{n}\right|^{2}\right]\left[\varphi_{n-1}^{*} \psi_{n-1}(z)+\varphi_{n-1}(z) \psi_{n-1}^{*}(z)\right] .
\end{aligned}
$$

Repeating the process and taking into account that

$$
\varphi_{0}^{*} \psi_{0}+\varphi_{0} \psi_{0}^{*}=2
$$


we then obtain

$$
\begin{aligned}
\varphi_{n}^{*}(z) & \psi_{n}(z)+\varphi_{n}(z) \psi_{n}^{*}(z) \\
= & 2 \frac{\kappa_{n}^{2}}{\kappa_{0}^{2}} \prod_{m=1}^{n} \frac{\overline{\alpha_{n}}}{\left|\alpha_{n}\right|}\left[\left.\varepsilon_{m}\right|^{2}-\left|\delta_{m}\right|^{2}\right] \frac{(-z)}{\left(1-\overline{\alpha_{n}} z\right)^{2}} \prod_{m=1}^{n-1} \frac{\left(\alpha_{m}-z\right)}{\left(1-\overline{\alpha_{m}} z\right)} .
\end{aligned}
$$

Multiplication by $\left(\alpha_{n}-z\right)$ in numerator and denominator then gives (7.1). (See also [4, p. 45].)

We recall that we may write $(\mathrm{cf} .(2.16))$

$$
\Phi_{n}=B_{n}+a_{n-1} B_{n-1}+\cdots+a_{0} B_{0}
$$

and hence

$$
\varphi_{n}=\kappa_{n} B_{n}+b_{n-1} B_{n-1}+\cdots+b_{0} B_{0}
$$

where $b_{k}=\kappa_{n} a_{k}$. It follows by orthogonality that

$$
\begin{gathered}
\left\langle\Phi_{n}, B_{n}\right\rangle=\left\langle\Phi_{n}, \Phi_{n}\right\rangle=\frac{1}{\kappa_{n}^{2}}, \\
\left\langle\varphi_{n}, B_{n}\right\rangle=\frac{1}{\kappa_{n}} .
\end{gathered}
$$

We also recall $(\mathrm{cf} .(2.15))$ that

$$
\left\langle\varphi_{n}^{*}, \zeta_{n}(z) P(z)\right\rangle=0 \text { when } P \in \mathscr{L}_{n-1} \text {. }
$$

Lemma 7.2. The following formula is valid:

$$
\frac{\kappa_{n-1}}{\kappa_{n}^{2}}=\frac{1}{\overline{\varepsilon_{n}}}\left[\left|\varepsilon_{n}\right|^{2}-\left|\delta_{n}\right|^{2}\right]\left\langle\frac{z-\alpha_{n-1}}{1-\overline{\alpha_{n}} z} \varphi_{n-1}, B_{n}\right\rangle .
$$

Proof. This follows by scalar multiplication of (4.7) by $B_{n}$, and use of (7.8) and (7.9).

Lemma 7.3. In the expansion (7.6) the coefficient $b_{n-1}$ has the value

$$
b_{n-1}=\left[\kappa_{n}-\overline{\varphi_{n}^{*}\left(\alpha_{n-1}\right)}\right] \frac{\overline{\alpha_{n}}}{\left|\alpha_{n}\right|} \frac{1-\overline{\alpha_{n-1}} \alpha_{n}}{\overline{\alpha_{n-1}}-\overline{\alpha_{n}}}
$$

when $\alpha_{n-1} \neq \alpha_{n}$.

Proof. We may write (7.6) as

$$
\varphi_{n}(z)=\kappa_{n} B_{n}(z)+b_{n-1} B_{n-1}(z)+L_{n-2}(z),
$$

where $L_{n-2} \in \mathscr{L}_{n-2}$. By taking the superstar transform of this equation we get

$$
\varphi_{n}^{*}(z)=\kappa_{n}+\overline{b_{n-1}} \zeta_{n}(z)+\zeta_{n}(z) \zeta_{n-1}(z) B_{n-2}(z)\left[L_{n-2}(z)\right]_{*} .
$$

Note that $\zeta_{n-1}\left(\alpha_{n-1}\right)=0$. Therefore

$$
\varphi_{n}^{*}\left(\alpha_{n-1}\right)=\kappa_{n}+\overline{b_{n-1}} \zeta_{n}\left(\alpha_{n-1}\right)
$$

hence (when $\alpha_{n-1} \neq \alpha_{n}$ )

$$
\overline{b_{n-1}}=\left[\varphi_{n}^{*}\left(\alpha_{n-1}\right)-\kappa_{n}\right] \frac{\left|\alpha_{n}\right|}{\overline{\alpha_{n}}} \frac{1-\alpha_{n-1} \overline{\alpha_{n}}}{\alpha_{n}-\alpha_{n-1}}
$$

from which (7.11) follows. 
Proposition 7.4. The coefficient sequences $\left\{\kappa_{n}\right\},\left\{\delta_{n}\right\},\left\{\varepsilon_{n}\right\}$ are connected through the formula

$$
\frac{1-\left|\alpha_{n}\right|^{2}}{1-\left|\alpha_{n-1}\right|^{2}} \cdot \frac{\kappa_{n-1}^{2}}{\kappa_{n}^{2}}=\left|\varepsilon_{n}\right|^{2}-\left|\delta_{n}\right|^{2}
$$

Proof. By the Hermitian property of the inner product we have

$$
\left\langle\frac{z-\alpha_{n-1}}{1-\overline{\alpha_{n}} z} \varphi_{n-1}, B_{n}\right\rangle=\left\langle\varphi_{n-1}, \frac{1-\overline{\alpha_{n-1}} z}{z-\alpha_{n}} B_{n}\right\rangle \text {. }
$$

We observe that we may write

$$
\frac{1-\overline{\alpha_{n-1}} z}{z-\alpha_{n}} B_{n}(z)=\frac{\overline{\alpha_{n}}-\overline{\alpha_{n-1}}}{1-\left|\alpha_{n}\right|^{2}} B_{n}(z)-\frac{\overline{\alpha_{n}}}{\left|\alpha_{n}\right|} \frac{\left(1-\overline{\alpha_{n-1}} \alpha_{n}\right)}{1-\left|\alpha_{n}\right|^{2}} B_{n-1}(z) .
$$

From (7.12) we then get

$$
\begin{aligned}
\frac{1-\overline{\alpha_{n-1}} z}{z-\alpha_{n}} B_{n}(z)= & \frac{\bar{\alpha}_{n}-\overline{\alpha_{n-1}}}{\kappa_{n}\left(1-\left|\alpha_{n}\right|^{2}\right)}\left[\varphi_{n}(z)-b_{n-1} B_{n-1}(z)-L_{n-2}(z)\right] \\
& -\frac{\overline{\alpha_{n}}}{\left|\alpha_{n}\right|} \frac{\left(1-\overline{\alpha_{n-1}} \alpha_{n}\right)}{\left(1-\left|\alpha_{n}\right|^{2}\right)} B_{n-1}(z)
\end{aligned}
$$

and by substitution for $b_{n-1}$ from (7.11) when $\alpha_{n-1} \neq \alpha_{n}$ this gives

$$
\begin{aligned}
& \frac{1-\overline{\alpha_{n-1}} z}{z-\alpha_{n}} B_{n}(z)=\frac{\bar{\alpha}_{n}-\overline{\alpha_{n-1}}}{\kappa_{n}\left(1-\left|\alpha_{n}\right|^{2}\right)} \varphi_{n}(z) \\
& \quad-\frac{\overline{\alpha_{n}}\left(1-\overline{\alpha_{n-1}} \alpha_{n}\right) \overline{\varphi_{n}^{*}\left(\alpha_{n-1}\right)}}{\left|\alpha_{n}\right| \kappa_{n}\left(1-\left|\alpha_{n}\right|^{2}\right)} B_{n-1}(z)-\frac{\bar{\alpha}_{n}-\overline{\alpha_{n-1}}}{\kappa_{n}\left(1-\left|\alpha_{n}\right|^{2}\right)} L_{n-2}(z)
\end{aligned}
$$

For $\alpha_{n-1}=\alpha_{n}$ this formula is immediately verified. (Recall that $\overline{\varphi_{n}^{*}\left(\alpha_{n-1}\right)}=$ $\overline{\varphi_{n}^{*}\left(\alpha_{n}\right)}=\kappa_{n}$.)

Scalar multiplication of (7.20) by $\varphi_{n-1}$, taking into account (7.8) for index $n-1$, then yields

$$
\left\langle\varphi_{n-1}, \frac{1-\overline{\alpha_{n-1}} z}{z-\alpha_{n}} B_{n}\right\rangle=\frac{-\alpha_{n} \varphi_{n}^{*}\left(\alpha_{n-1}\right)\left(1-\alpha_{n-1} \overline{\alpha_{n}}\right)}{\left|\alpha_{n}\right| \kappa_{n}\left(1-\left|\alpha_{n}\right|^{2}\right) \kappa_{n-1}}
$$

and consequently by (7.17) we get

$$
\left\langle\frac{z-\alpha_{n-1}}{1-\bar{\alpha}_{n} z} \varphi_{n-1}, B_{n}\right\rangle=\frac{-\alpha_{n} \varphi_{n}^{*}\left(\alpha_{n-1}\right)\left(1-\alpha_{n-1} \bar{\alpha}_{n}\right)}{\left|\alpha_{n}\right| \kappa_{n}\left(1-\left|\alpha_{n}\right|^{2}\right) \kappa_{n-1}} .
$$

Substituting (7.22) for $\left\langle\frac{z-\alpha_{n-1}}{1-\overline{\alpha_{n} z} z} \varphi_{n-1}, B_{n}\right\rangle$ and (4.5) for $\varepsilon_{n}$ in (7.10) we obtain (7.16).

Corollary 7.5. The coefficient sequences $\left\{\kappa_{n}\right\},\left\{\delta_{n}\right\},\left\{\varepsilon_{n}\right\}$ are connected through the formula

$$
\frac{\kappa_{0}^{2}}{\kappa_{n}^{2}}\left(1-\left|\alpha_{n}\right|^{2}\right)=\prod_{m=1}^{n}\left[\left|\varepsilon_{m}\right|^{2}-\left|\delta_{m}\right|^{2}\right]
$$

Proof. Follows by multiplication of the equalities (7.16) for indices $1,2, \ldots$, $n$. 
We recall the inequality between weighted geometric and arithmetic means, see, e.g. $[20,36]$ :

$$
\exp \left(\frac{1}{P} \int_{-\pi}^{\pi} p(\theta) \ln f(\theta) d \theta\right) \leq \frac{1}{P} \int_{-\pi}^{\pi} p(\theta) f(\theta) d \theta
$$

where $p(\theta) \geq 0$ and integrable, $P=\int_{-\pi}^{\pi} p(\theta) d \theta, f(\theta) \geq 0$. With $p(\theta)=p_{n}(\theta)$ this in particular gives

$$
\exp \left(\frac{1}{2 \pi} \int_{-\pi}^{\pi} p_{n}(\theta) \ln f(\theta) d \theta\right) \leq \frac{1}{2 \pi} \int_{\pi}^{\pi} p_{n}(\theta) f(\theta) d \theta .
$$

Proposition 7.6. The following inequality holds:

$$
\kappa_{n}^{2} \leq \frac{1}{2 \pi} \exp \left(-\frac{1}{2 \pi} \int_{-\pi}^{\pi} p_{n}(\theta) \ln \left[\frac{\mu^{\prime}(\theta)}{p_{n}(\theta)}\right] d \theta\right) .
$$

Proof. Since $\int_{-\pi}^{\pi}\left|\varphi_{n}^{*}\left(e^{i \theta}\right)\right|^{2} d \mu(\theta)=1$ and $\int_{-\pi}^{\pi} g(\theta) d \mu(\theta) \geq \int_{-\pi}^{\pi} g(\theta) \mu^{\prime}(\theta) d \theta$ for $g(\theta) \geq 0$, we obtain

$$
1 \geq \int_{-\pi}^{\pi}\left|\varphi_{n}^{*}\left(e^{i \theta}\right)\right|^{2} \mu^{\prime}(\theta) d \theta=\int_{-\pi}^{\pi} p_{n}(\theta)\left|\varphi_{n}^{*}\left(e^{i \theta}\right)\right|^{2}\left[\frac{\mu^{\prime}(\theta)}{p_{n}(\theta)}\right] d \theta .
$$

Using (7.25) we then get

$$
\begin{aligned}
1 & \geq 2 \pi \exp \left(\frac{1}{2 \pi} \int_{-\pi}^{\pi} p_{n}(\theta) \ln \left(\left|\varphi_{n}^{*}\left(e^{i \theta}\right)\right|^{2}\left[\frac{\mu^{\prime}(\theta)}{p_{n}(\theta)}\right]\right) d \theta\right) \\
& =2 \pi \exp \left(\frac{1}{2 \pi} \int_{-\pi}^{\pi} p_{n}(\theta) \ln \left|\varphi_{n}^{*}\left(e^{i \theta}\right)\right|^{2} d \theta\right) \cdot \exp \left(\frac{1}{2 \pi} \int_{-\pi}^{\pi} p_{n}(\theta) \ln \left[\frac{\mu^{\prime}(\theta)}{p_{n}(\theta)}\right] d \theta\right) .
\end{aligned}
$$

Since $\ln \left|\varphi_{n}^{*}(z)\right|^{2}$ is harmonic for $z \in \mathbf{D} \cup \mathbf{T}$,

$$
\frac{1}{2 \pi} \int_{-\pi}^{\pi} p_{n}(\theta) \ln \left|\varphi_{n}^{*}\left(e^{i \theta}\right)\right|^{2} d \theta=\ln \left|\varphi_{n}^{*}\left(\alpha_{n}\right)\right|^{2} .
$$

The inequality (7.28) may then be written

$$
1 \geq 2 \pi\left|\varphi_{n}^{*}\left(\alpha_{n}\right)\right|^{2} \exp \left(\frac{1}{2 \pi} \int_{-\pi}^{\pi} p_{n}(\theta) \ln \left[\frac{\mu^{\prime}(\theta)}{p_{n}(\theta)}\right] d \theta\right),
$$

which is equivalent to (7.26).

We shall use the notations

$$
\begin{gathered}
\beta_{n-1}^{(n)}=\left\langle\frac{1-\overline{\alpha_{n-1}} z}{z-\alpha_{n}}, \varphi_{n-1}(z)\right\rangle, \\
\gamma_{n-1}^{(n)}=\left\langle\frac{1-\overline{\alpha_{n-1}} z}{1-\overline{\alpha_{n}} z}, \varphi_{n-1}(z)\right\rangle .
\end{gathered}
$$

Lemma 7.7. The following equality holds:

$$
\gamma_{n-1}^{(n)}=-\frac{\left|\alpha_{n}\right|}{\overline{\alpha_{n}}} \frac{\varepsilon_{n}}{\kappa_{n-1}}, \quad n=1,2, \ldots
$$

Proof. By definition $\varphi_{n-1}^{*}(z)=B_{n-1}(z)\left(\varphi_{n-1}\right)_{*}(z)$, hence

$$
\gamma_{n-1}^{(n)}=\left\langle\frac{1-\overline{\alpha_{n-1}} z}{1-\overline{\alpha_{n}} z} B_{n-1}(z), \varphi_{n-1}(z)\right\rangle .
$$


We observe that we may write

$$
\frac{1-\overline{\alpha_{n-1}} z}{1-\overline{\alpha_{n}} z} B_{n-1}(z)=\frac{1-\overline{\alpha_{n-1}} \alpha_{n}}{1-\left|\alpha_{n}\right|^{2}} B_{n-1}(z)+\frac{\left|\alpha_{n}\right|}{\overline{\alpha_{n}}} \frac{\overline{\alpha_{n-1}}-\overline{\alpha_{n}}}{1-\left|\alpha_{n}\right|^{2}} B_{n}(z) .
$$

By using (7.6), (7.34), and Lemma 7.3 we then get

$$
\frac{1-\overline{\alpha_{n-1}} z}{1-\overline{\alpha_{n}} z} B_{n-1}(z)=\frac{\left(1-\overline{\alpha_{n-1}} \alpha_{n}\right) \overline{\varphi_{n}^{*}\left(\alpha_{n-1}\right)}}{\left(1-\left|\alpha_{n}\right|^{2}\right) \kappa_{n} \kappa_{n-1}} \varphi_{n-1}(z)+c_{1} \varphi_{n}(z)+c_{2} L_{n-2}(z),
$$

where $L_{n-2} \in \mathscr{L}_{n-2}, c_{1}$, and $c_{2}$ are constants.

(In Lemma 7.3 it is assumed that $\alpha_{n-1} \neq \alpha_{n}$. If $\alpha_{n-1}=\alpha_{n}$, the result follows immediately, with $c_{1}=0$.)

From (7.33) we then get

$$
\gamma_{n-1}^{(n)}=\frac{\left(1-\overline{\alpha_{n-1}} \alpha_{n}\right) \overline{\varphi_{n}^{*}\left(\alpha_{n-1}\right)}}{\left(1-\left|\alpha_{n}\right|^{2}\right) \kappa_{n} \kappa_{n-1}},
$$

and by (4.5) the right-hand side equals $-\frac{\left|\alpha_{n}\right|}{\bar{\alpha}_{n}} \frac{\varepsilon_{n}}{\kappa_{n-1}}$.

Lemma 7.8. The following equality holds:

$$
\beta_{n-1}^{(n)}=\frac{\bar{\alpha}_{n}}{\left|\alpha_{n}\right|} \frac{\overline{\delta_{n}}}{\kappa_{n-1}}, \quad n=1,2, \ldots
$$

Proof. We may write

$$
\overline{\beta_{n-1}^{(n)}}=\left\langle\frac{z-\alpha_{n-1}}{1-\overline{\alpha_{n}} z} \varphi_{n-1}(z), 1\right\rangle .
$$

Scalar multiplication of (4.1) by 1 then gives

$$
0=\varepsilon_{n} \frac{\kappa_{n}}{\kappa_{n-1}} \overline{\beta_{n-1}^{(n)}}+\delta_{n} \frac{\kappa_{n}}{\kappa_{n-1}} \gamma_{n-1}^{(n)},
$$

hence by (7.32)

$$
\overline{\beta_{n-1}^{(n)}}=\frac{\left|\alpha_{n}\right| \delta_{n}}{\overline{\alpha_{n}} \kappa_{n-1}},
$$

which is equivalent to (7.37).

Lemma 7.9. The following equality holds:

$$
\begin{aligned}
& \sum_{m=1}^{n}\left|\beta_{m-1}^{(m)}\right|^{2}\left(1-\left|\alpha_{m-1}\right|^{2}\right) \\
& \quad=\frac{1-\left|\alpha_{0}\right|^{2}}{\kappa_{0}^{2}}-\frac{1-\left|\alpha_{n}\right|^{2}}{\kappa_{n}^{2}}+\sum_{m=1}^{n} \frac{\left(1-\left|\alpha_{m-1}\right|^{2}\right)}{\kappa_{m-1}^{2}}\left(\left|\varepsilon_{m}\right|^{2}-1\right) .
\end{aligned}
$$

Proof. From (7.37) we get

$$
\left|\beta_{m-1}^{(m)}\right|^{2}=\frac{\left|\delta_{m}\right|^{2}}{\kappa_{m-1}^{2}}
$$

and from (7.16) we get

$$
\left(1-\left|\alpha_{m-1}\right|^{2}\right) \frac{\left|\delta_{m}\right|^{2}}{\kappa_{m-1}^{2}}=\left(1-\left|\alpha_{m-1}\right|^{2}\right) \frac{\left|\varepsilon_{m}\right|^{2}}{\kappa_{m-1}^{2}}-\frac{1-\left|\alpha_{m}\right|^{2}}{\kappa_{m}^{2}} .
$$


The right-hand side of (7.43) may be written

$$
\begin{aligned}
& \left(1-\left|\alpha_{m-1}\right|^{2}\right) \frac{\left|\varepsilon_{m}\right|^{2}}{\kappa_{m-1}^{2}}-\frac{1-\left|\alpha_{m}\right|^{2}}{\kappa_{m}^{2}} \\
& \quad=\frac{1-\left|\alpha_{m-1}\right|^{2}}{\kappa_{m-1}^{2}}-\frac{1-\left|\alpha_{m}\right|^{2}}{\kappa_{m}^{2}}+\frac{\left(1-\left|\alpha_{m-1}\right|^{2}\right)}{\kappa_{m-1}^{2}}\left(\left|\varepsilon_{m}\right|^{2}-1\right) .
\end{aligned}
$$

Consequently by summing (7.43) for $m=1,2, \ldots, n$ we get (7.41).

\section{REFERENCES}

1. N. I Akhiezer, The classical moment problem and some related questions in analysis, Hafner, New York, 1965.

2. A. Bultheel, On a special Laurent-Hermite interpolation problem, Numerische Methoden der Approximations theorie 6 (L. Collatz, G. Meinardus, and H. Werner, eds.), Birkhäuser, Basel, 1981, pp. 63-79.

3. A. Bultheel and P. Dewilde, Orthogonal functions related to the Nevanlinna-Pick problem, Mathematical Theory of Networks and Systems, Proc. MTNS Conf., Delft, The Netherlands (P. Dewilde, ed.), Western Periodicals, North Hollywood, 1979, pp. 207-211.

4. A. Bultheel, P. Gonzalez-Vera, E. Hendriksen, and O. Njàstad, A. Szegö theory for rational functions, Technical Report TW-131, K.U. Leuven, Dept. of Comput. Sci., May 1990.

5. __ Orthogonal rational functions similar to Szegö polynomials, IMACS Annals of Computing and Applied Mathematics (C. Brezinski, L. Gori et al., eds.), Baltzer AG, Basel, 1991, pp. 195-204.

6. The computation of orthogonal rational functions and their interpolating properties, Numerical Algorithms 2 (1992), 85-114.

7. _ A Favard theorem for orthogonal rational functions on the unit circle, Numerical Algorithms 3 (1992), 81-90.

8. rithms 3 (1992), 105-116.

9. _ـ A moment problem associated to rational Szegö functions, Numerical Algorithms 3 (1992), 91-104.

10. 49-68.

11. (1994), 221-243.

12. - Recurrence relations for orthogonal functions, Continued Fractions and Orthogonal Functions (S. C. Cooper and W. J. Thron, eds.), Marcel Dekker, 1994, pp. 23-46.

13. C. Carathéodory, Über den Variabilitätsbereich der Koeffizienten von Potenzreihen die gegebene Werte nicht annehmen, Math. Ann. 64 (1907), 95-115.

14. P. Delsarte, Y. Genin, and Y. Kamp, On the role of the Nevanlinna-Pick problem in circuit and system theory, Internat. J. Circuit Theory Appl. 9 (1981), 177-187.

15. P. Delsarte and Y. Genin, On the role of orthogonal polynomials on the unit circle in digital signal processing applications, Orthogonal Polynomials: Theory and Practice (P. Nevai, ed.), Kluwer, Dordrecht, Boston, and London, 1990, pp. 115-133.

16. P. Dewilde and H. Dym, Schur recursion, error formulas, and convergence of rational estimators for stationary stochastic sequences, IEEE Trans. Inform. Theory 27 (1981), 446-461.

17. M. M. Djrbashian, A survey on the theory of orthogonal systems and some open problems, Orthogonal Polynomials: Theory and Practice (P. Nevai, ed.), Kluwer, Dordrecht, Boston, and London, 1990, pp. 135-146.

18. P. L. Duren, Theory of $H^{P}$ spaces, Academic Press, New York, 1970.

19. Y. L. Geronimus, Polynomials orthogonal on a circle and their applications, Amer. Math. Soc. Transl. 104 (1954), 79. 
20. __ Orthogonal polynomials, Consultants Bureau, New York, 1961.

21. P. Gonzalez-Vera and O. Njảstad, Szegö functions and multipoint Padé approximation, J. Comput. Appl. Math. 32 (1990), 107-116.

22. U. Grenander and G. Szegö, Toeplitz forms and their applications, Chelsea, Berkeley, 1958.

23. P. Henrici, Applied and computational complex analysis, Vols. 1 and 2, Wiley, New York, 1974 and 1977.

24. G. Herglotz, Über Potenzreihen mit positiven reellen Teil im Einheitskreis, Ber. Verh. Sächs, Ges. Wiss. Leipzig Math. Phys. Kl. 63 (1911), 501-511.

25. K. Hoffman, Banach spaces of analytic functions, Prentice-Hall, Englewood Cliffs, NJ, 1962.

26. W. B. Jones, O. Njảstad, and W. J. Thron, Moment theory, orthogonal polynomials, quadrature, and continued fractions associated with the unit circle, Bull. London Math. Soc. 21 (1989), 113-152.

27. W. B. Jones and O. Njảstad, Applications of Szegö polynomials to digital signal processing, Rocky Mountain J. Math. 21 (1991), 387-436.

28. M. G. Krein and A. A. Nudelman, The Markov moment problem and extremal problems, Transl. Math. Monos., No. 150, Amer. Math. Soc., Providence, RI, 1977.

29. H. J. Landau (ed.), Moments in mathematics, Proc. Sympos. Appl. Math., vol. 37, Amer. Math. Soc., Providence, RI, 1987.

30. G. Lopez, Conditions for convergence of multipoint Padé approximants for functions of Stieltjes type, Math. USSR-Sb. 35 (1979), 363-375.

31. _ Szegö's theorem for polynomials orthogonal with respect to varying measures, Orthogonal Polynomials and their Applications (Alfaro et al., eds.), Lecture Notes in Math., vol. 1329, Springer, Berlin, 1988, pp. 255-260.

32. R. Nevanlinna, Über beschränkte Functionen die in gegebenen Punkten vorgeschriebene Werte annehmen, Ann. Acad. Sci. Fenn. Sect. A 13 (1919), No. 1.

33. K. Pan, Strong and weak convergence of orthogonal systems of rational functions on the unit circle, J. Comput. Appl. Math. 46 (1993), 427-436.

34. _ On characterization theorems for measures associated with orthogonal systems of rational functions on the unit circle, J. Approx. Theory 70 (1992), 256-272.

35. G. Pick, Über die Beschränkungen analytischer Funktionen welche durch vorgegebene Functionswerte bewirkt werden, Math. Ann. 77 (1916), 7-23.

36. I. I. Priwalow, Randeigenschaften Analytischer Funktionen, VEB Deutscher-Verlag der Wissenschaften, Berlin, 1956.

37. W. Rudin, Real and complex analysis, 2nd ed., McGraw-Hill, New York, 1974.

38. G. Szegö, Orthogonal polynomials, Amer. Math. Soc. Colloq. Publ., vol. 23, 4th ed., Amer. Math. Soc., Providence, RI, 1975.

Department of Computer Science, K. U. Leuven, B-3001 Leuven, Belgium

E-mail address: ade@c.s.kuleuven.ac.be

Facultad de Matemáticas, Universidad de la laguna, la laguna, Tenerife, Canary ISLANDS, SPAIN

Department of Mathematics, University of Amsterdam, Plantage Muidergracht 24, 1018 TV AMSTERDAM, THE NeTHERLANDS

E-mail address: erik@fwi.uva.nl

Department of Mathematical Sciences, University of Trondheim-NTH, N-7034 TrondHEIM, NORWAY

E-mail address: njastad@imf unit.no 